\title{
Metabolism of Phospholipids in Nocardia polychromogenes
}

\author{
By A. K. TRANA, G. K. KHULLER AND D. SUBRAHMANYAM \\ Department of Biochemistry, Postgradi:ate Institute of Medical Education \\ and Research, Chandigarh 160012, India
}

(Received 6 April 1979; revised 17 July 1979)

\begin{abstract}
The rates of synthesis and breakdown of phospholipids in growing cultures of Nocardia polychromogenes were investigated by means of a pulse-labelling technique using ${ }^{32} \mathrm{PO}_{4}{ }^{3-}$. The results indicated that phospholipids were broken down and phosphatidylinositol mannosides had a high turnover rate. The other two main components, cardiolipin and phosphatidylethanolamine, had a relatively low rate of turnover.
\end{abstract}

\section{INTRODUCTION}

Cardiolipin, phosphatidylethanolamine and phosphatidylinositol mannosides are the three major components of the phospholipids of Nocardia (Khuller, 1976, 1977). Phospholipids of Nocardia are antigenic in nature (Trana \& Khuller, 1977) and elicit antibodies in experimental nocardiosis (Trana et al., 1978). These observations have stimulated research on nocardial lipids.

Several reports have been published on the turnover rates of phosphatidylglycerol, cardiolipin and phosphatidylethanolamine in Escherichia coli (Kanemasa et al., 1967; Ballesta et al., 1973) and other bacteria (Short \& White, 1971; Subrahmanyam, 1965; Akamatsu et al., 1967), but no such studies have been reported with Nocardia. In this paper, the results of the incorporation of ${ }^{32} \mathrm{PO}_{4}{ }^{3-}$ into individual phospholipids are reported together with chase studies on the pre-labelled phospholipids in Nocardia polychromogenes.

\section{METHODS}

Bacterial strain and cultivation. Nocardia polychromogenes NRRL B-1531, originally obtained from Dr Y. Okami, Institute of Microbial Chemistry, Tokyo, Japan, was maintained on Lowenstein-Jensen medium (Rippon, 1974). Cells were grown in stationary culture in Sabouraud's dextrose broth at pH 7.0 and $37^{\circ} \mathrm{C}$.

Incorporation of ${ }^{32} \mathrm{PO}_{4}{ }^{3-}$ into phospholipids. Nocardia polychromogenes grown for $4 \mathrm{~d}$ in Sabouraud's medium was harvested by filtration under sterile conditions and washed with normal saline; about $10 \mathrm{~g}$ wet wt bacteria were resuspended in $100 \mathrm{ml}$ Sabouraud's medium minus peptone (this medium contains no phosphate). Carrier-free ${ }^{32} \mathrm{PO}_{4}{ }^{3-}(5 \mathrm{mCi})$ in dilute $\mathrm{HCl}$ solution (obtained from Bhabha Atomic Research Centre, Bombay, India) was added to the suspension and incubation was continued at $37{ }^{\circ} \mathrm{C}$. At intervals, the cells were collected by filtration and phospholipids were extracted and examined as described below.

The radioactivity of each sample was counted in a Packard liquid scintillation counter using a toluenebased scintillation fluid containing $0.4 \%(\mathrm{w} / \mathrm{v})$ 2,5-diphenyloxazole and $0.05 \%(\mathrm{w} / \mathrm{v})$ 1,4-di-2-(5-phenyloxazolyl)benzene.

Loss of radioactivity from pulse-labelled phospholipids. The culture was labelled by exposure to ${ }^{32} \mathrm{PO}_{4}{ }^{3-}$ for $6 \mathrm{~h}$ as described above. Labelled cells were collected by filtration, washed with normal saline, resuspended in normal Sabouraud's medium containing $0.013 \%(\mathrm{w} / \mathrm{v})$ unlabelled phosphate $\left(\right.$ as $\left.\mathrm{P}_{2} \mathrm{O}_{5}\right)$ and incubated at $37^{\circ} \mathrm{C}$. The cells were harvested after subsequent growth for 0 to $24 \mathrm{~h}$, within the exponential growth phase. The lipids were extracted as described below and the radioactivity of each sample was measured as described above.

Extraction of lipids. Cells were suspended in chloroform/methanol $(2: 1 \mathrm{v} / \mathrm{v})$ and stirred with a magnetic stirrer for $4 \mathrm{~h}$. The homogenate was filtered and the filtrate was washed as described by Folch et al. (1957). 


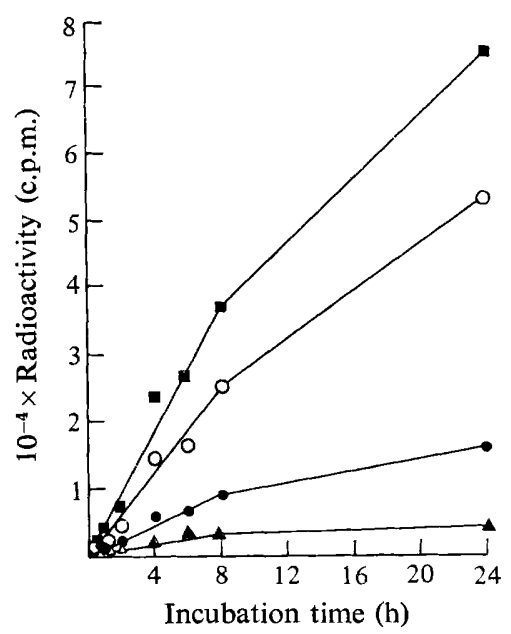

Fig. 1

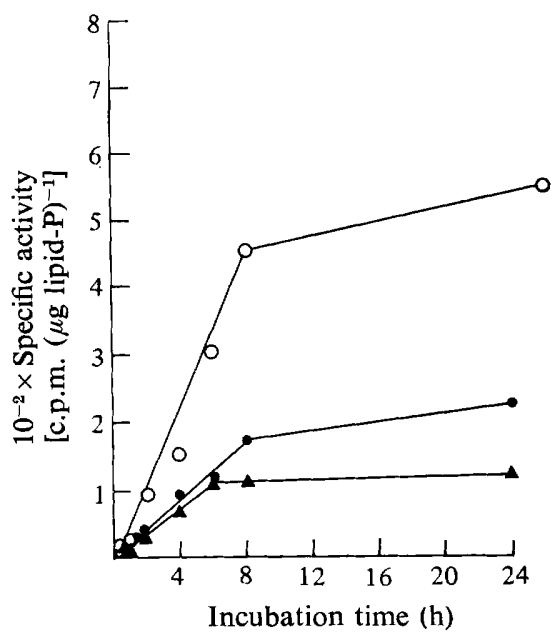

Fig. 2

Fig. 1. ${ }^{32} \mathrm{PO}_{4}{ }^{3-}$ incorporation into total phospholipids ( $\square$ ) and into individual phospholipids of $N$. polychromogenes: phosphatidylinositol mannosides $(\bigcirc)$, cardiolipin $(\bigcirc)$ and phosphatidylethanolamine $(\mathbf{\Delta})$.

Fig. 2. Changes in the specific activities of individual phospholipids of $N$. polychromogenes after ${ }^{32} \mathrm{PO}_{4}{ }^{3-}$ incorporation: phosphatidylinositol mannosides (O), cardiolipin (O) and phosphatidylethanolamine $(\mathbf{\Delta})$.

Chromatographic separation of phospholipids and their quantitative analysis. The separation, isolation, characterization and measurement of phospholipids were carried out as described by Khuller (1977). In addition, the spots corresponding to each component were scraped from the chromatographic plates and their radioactivity was measured.

\section{RESULTS}

Incorporation of ${ }^{32} \mathrm{PO}_{4}{ }^{3-}$ into individual phospholipids. The rate at which ${ }^{32} \mathrm{PO}_{4}{ }^{3-}$ was incorporated into the total phospholipids of $N$. polychromogenes was linear for the first $8 \mathrm{~h}$ (Fig. 1) and continued to increase significantly up to $24 \mathrm{~h}$. The radioactivities of individual phospholipids were measured after thin-layer chromatography: phosphatidylinositol mannosides had the highest total radioactivity and phosphatidylethanolamine the lowest (Fig. 1). The highest specific radioactivity was found in the phosphatidylinositol mannosides and the lowest in phosphatidylethanolamine (Fig. 2).

Turnover of phospholipids. The breakdown of phospholipids within the cell was studied by chasing the pre-labelled phospholipids with unlabelled phosphate (Fig. 3). The radioactivity of the phospholipids continued to decrease for $18 \mathrm{~h}$; subsequently, there was a slight increase when incubation was continued up to $24 \mathrm{~h}$. The loss of radioactivity indicated that phospholipids were broken down in growing cells.

The radioactivity of the individual components of the phospholipids was measured to determine their turnover (Fig. 3). Phosphatidylinositol mannosides showed the highest rate of degradation of the three components studied. Cardiolipin showed no appreciable change in radioactivity for the first $6 \mathrm{~h}$ but exhibited a slight increase during the next $18 \mathrm{~h}$. There was no loss of radioactivity from phosphatidylethanolamine, indicating that no turnover occurred. The specific radioactivities of the individual phospholipids after chasing (Fig. 4) revealed the same pattern indicating that the phosphatidylinositol mannosides had a high rate of turnover within growing cells while the other two components showed no noticeable turnover. 


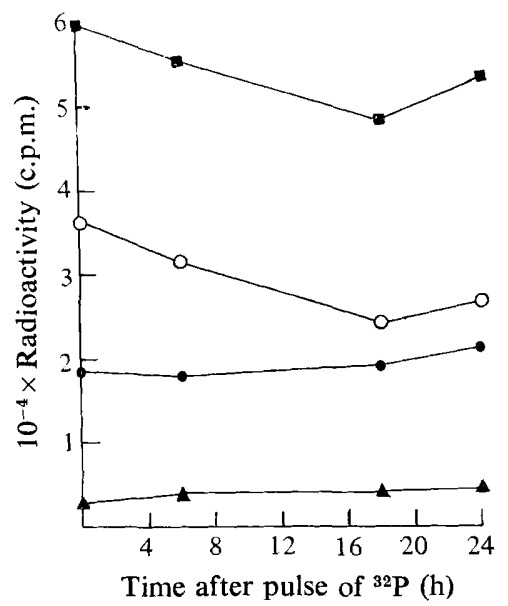

Fig. 3

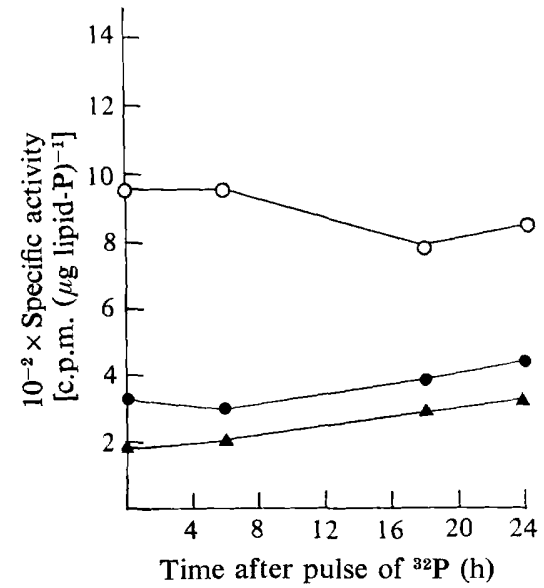

Fig. 4

Fig. 3. Loss of radioactivity from the total phospholipid fraction ( $\boldsymbol{a})$ of $N$. polychromogenes after pulse labelling and from individual phospholipids: phosphatidylinositol mannosides $(O)$, cardiolipin $(\boldsymbol{O})$ and phosphatidylethanolamine $(\boldsymbol{\Delta})$.

Fig. 4. Loss of specific activity from individual phospholipids of $N$. polychromogenes after pulse labelling: phosphatidylinositol mannosides $(0)$, cardiolipin $(\circlearrowleft)$ and phosphatidylethanolamine (A).

\section{DISCUSSION}

In bacteria, as in higher organisms, phospholipids are mainly found in the membranes (Lennarz, 1972) and have a major role in their structural and functional organization (Cronan, 1978; Singer \& Nicolson, 1972). The study of phospholipid metabolism in general is therefore of immense importance in the elucidation of the role of these components in the membranes.

The radioactivity of the total phospholipids increased at a linear rate up to $8 \mathrm{~h}$ in $N$. polychromogenes. This compares with linear rates of incorporation of ${ }^{32} \mathrm{PO}_{4}{ }^{3-}$ into the total phospholipids of Mycobacterium 607 (Subrahmanyam, 1965) and Mycobacterium phlei (Akamatsu et al., 1967) of $30 \mathrm{~min}$ and $2 \mathrm{~h}$, respectively. The radioactivity of the individual phospholipids in $N$. polychromogenes revealed that the rate of biosynthesis of phosphatidylinositol mannosides is the highest of the three components studied, in contrast to the results obtained with $M$. phlei (Akamatsu et al., 1967) where the maximum incorporation was into cardiolipin.

Studies on the metabolism of phospholipids of different bacteria (Ballesta et al., 1973; Dhariwal et al., 1978; Short \& White, 1971) have indicated a high turnover rate. The results of the present study demonstrate that the phospholipids of $N$. polychromogenes also undergo turnover. Phosphatidylinositol mannosides showed a rapid turnover whereas cardiolipin and phosphatidylethanolamine had relatively low rates of turnover. This turnover of phosphatidylinositol mannosides agrees with the results obtained with Mycobacterium 607 (Subrahmanyam, 1965) but contrasts with those obtained with M. phlei (Akamatsu et al., 1967; Dhariwal et al., 1978), where phosphatidylinositol mannosides have a low turnover rate as they probably form part of the cell envelope (Akamatsu et al., 1966). It is, however, difficult to explain the rapid loss of radioactivity from phosphatidylinositol mannosides without knowing the role of these components in the structure and metabolism of N. polychromogenes. The slow turnover of cardiolipin differs from that in mycobacteria (Akamatsu et al., 1967; Dhariwal et al., 1978) where it has the highest turnover.

The third component of the phospholipids of $N$. polychromogenes, phosphatidylethanolamine, did not undergo turnover during growth, suggesting that once formed, it is completely 
stable. These results agree with those reported for $E$. coli (Kanfer \& Kennedy, 1963) and M. phlei (Akamatsu et al., 1967; Dhariwal et al., 1978).

We wish to acknowledge the contributions of Miss Shobha Bhargava in the initial part of this work.

\section{REFERENCES}

Akamatsu, Y., ONo, Y. \& Nojima, S. (1966). Phospholipid patterns in subcellular fractions of Mycobacterium phlei. Journal of Biochemistry 59, $176-182$.

Akamatsu, Y., Ono, Y. \& Nojima, S. (1967). Studies on the metabolism of phospholipids in Mycobacterium phlei. Journal of Biochemistry 61, 96-102.

Ballesta, J.P. G., De-Garcia, C. L. \& Schaechter, M. (1973). Turnover of phosphatidyl glycerol in Escherichia coli. Journal of Bacteriology 116, 210-214.

Cronan, J. E. (1978). Molecular biology of bacterial membrane lipids. Annual Review of Biochemistry 47, 163-189.

Dhariwal, K. R., Chander, A. \& Subramanian, T. A. V. (1978). Turnover of lipids in Mycobacterium smegmatis CDC 46 and Mycobacterium phlei ATCC 354. Archives of Microbiology 116, 69-75.

Folch, J., Lees, M. \& Stanley, G. H. S. (1957). A simple method for the isolation and purification of total lipids from animal tissues. Journal of Biological Chemistry 226, 497-509.

Kanemasa, Y., Akamatsu, Y. \& Nojima, S. (1967). Composition and turnover of the phospholipids in Escherichia coli. Biochimica et biophysica acta 144, 382-390.

KANFER, J. \& KenNedy, E. P. (1963). Metabolism of phospholipids in Escherichia coli B. Journal of Biological Chemistry 238, 2919-2922.
KHULlER, G. K. (1976). The mannophosphoinositides of Nocardia asteroides. Experientia 32, 1371-1372.

KhUller, G. K. (1977). Phospholipid composition of Nocardia species. Indian Journal of Medical Research 65, 657-660.

LenNarz, W. J. (1972). Studies on the biosynthesis and functions of lipids in bacterial membranes. Accounts of Chemical Research 5, 361-367.

RIPPON, J. W. (1974). Medical Mycology, pp. 34-40. Philadelphia: W. B. Saunders.

SHORT, S. A. \& WHITE, D. C. (1971). Metabolism of phosphatidyl glycerol, lysophosphatidyl glycerol and cardiolipin of Staphylococcus aureus. Journal of Bacteriology 108, 219-226.

Singer, S. J. \& Nicolson, G. L. (1972). The fluid mosaic model of the structure of cell membranes. Science 175, 720-731.

Subrahmanyam, D. (1965). Studies on the metabolism of phosphatides of Mycobacterium 607. Indian Journal of Biochemistry 2, 27-30.

Trana, A. K. \& Khuller, G. K. (1977). Immunochemical studies with phosphatides of Nocardia. Indian Journal of Biochemistry and Biophysics 14, 385-386.

Trana, A. K., Sehgal, S. C. \& Khuller, G. K. (1978). Antibodies to phospholipids in experimental nocardiosis. Antonie van Leeuwenhoek 44, 391-394. 